\title{
Editorial
}

\section{Efectos de la migración Ngöbe Buglé en el Servicio de Pediatría del Hospital Dr. Maximiliano Peralta de Cartago}

Desde hace muchos años el personal del Servicio de Pediatría del Hospital Dr. Maximiliano Peralta, enfrenta otra cara de la realidad: las migraciones laborales de indígenas procedentes de la comarca Ngöbe Buglé de Panamá, y que se desplazan año con año hacia la zona de los Santos de San Marcos de Tarrazú para trabajar en la recolección de café. Por tal motivo, durante el periodo comprendido entre los meses de octubre a marzo de cada año la Clínica de los Santos, refiere a este centro indígenas inmigrantes para ser hospitalizados.

En el informe "estudio de la migraciones internacionales", publicado por la Oficina Internacional del trabajo de Ginebra, en el año 2002; se menciona que los pobladores de la comarca Ngöbe Buglé emigran hacia Costa Rica con el fin de obtener dinero para resolver sus necesidades básicas de alimentación, situación que nos enfrenta con la pobreza extrema. La pobreza indígena en Panamá es catalogada de "abismal" por el Banco Mundial y de "masiva y profunda" por el Gobierno Nacional de Panamá.

De acuerdo al análisis de las carencias en dimensiones fundamentales para el desarrollo humano, que se desprende del índice de pobreza humana, los Ngöbe Buglé ocupan el primer lugar en la escala de pobreza, y que por ende son los indígenas más pobres entre los pobres.

Esta población indígena presenta cifras altas de mortalidad materna e infantil, analfabetismo, desnutrición, carencia de servicios básicos: de agua, alimentación e ingresos mínimos y los menores de 6 a 9 años están desnutridos en un $71.8 \%$.

La migración les proporcionan ingresos mínimos de subsistencia mientras están fuera de su país pues mitiga el hambre, también, los hace más vulnerables a otros hábitos con el alcohol y las drogas. En época de recolección de café se hace visible el drama de centenares de Ngöbe embriagados y tirados en el suelo, o peleando con otros. Debido a que las mujeres y los niños no disponen de dinero sino que este es administrado por el hombre (aunque la mujer haya trabajado igual que él), esto afecta la armonía familiar y la alimentación de los niños. La mujer Ngöbe sufre mucha discriminación, trabajan mucho y su trabajo no es reconocido; muchas veces no son tomadas en cuenta en las decisiones relacionadas con el desarrollo familiar están expuestas en mayor grado que los hombres al analfabetismo y a la desnutrición.

La ideología del olvido, la marginación social y económica, que sufre este grupo poblacional, se deducen de los numerosos estudios sobre sus condiciones de vida, realizados por entidades oficiales, religiosas, organizaciones no gubernamentales y organizaciones internacionales.

A pesar de la cifra elevada de inmigrantes indígenas más de 5000 Ngöbes, que ingresan al país año con año, durante la recolección de café, existe un gran desconocimiento sobre las particularidades de estas migraciones laborales, asociado también con el carácter irregular de los movimientos, a que estas personas generalmente ingresan sin documentos y a la existencia de registro estadístico insuficientes que conllevan a un subregistro importante. 
De acuerdo al documento "Migraciones y desafíos de una política migratoria integral en Costa Rica" incluido en el libro "Sociedades hospitalarias: Costa Rica y la acogida de inmigrantes" el Sr. Abelardo Morales G, indica que Costa Rica no cuenta con información sobre perfiles socio demográficos de la inserción laboral de los panameños inmigrantes; y que de igual forma, las autoridades costarricenses relacionadas con esta temática tampoco disponen de información, debido a que este grupo no ha sido objeto de suficiente atención en el tratamiento de las políticas públicas.

Cada año durante los meses de octubre a marzo el Servicio de Pediatría del Hospital Dr. Maximiliano Peralta de Cartago, atiende a un grupo de pacientes y a su núcleo familiar culturalmente diferentes a la población del área de atracción del hospital, ya que no hablan español a excepción de algunos pocos, con hábitos diferentes y que tienen escasa comunicación con el personal del servicio, lo que dificulta el cumplimiento de las normas que regulan el trabajo diario de los funcionarios del hospital. Como medida de gestión el Servicio se ve obligado a tomar acciones para evitar infecciones intrahospitalarias y reingresos.

Estos niños gozan del derecho de admisión conjunta las $24 \mathrm{hrs}$ del día, se proporciona a las madres la alimentación y sillas necesarias para que puedan reposar en algún momento.

Debido a estas inmigraciones el perfil epidemiológico del Servicio cambia, todos estos pacientes tienen anemia, desnutrición y parasitosis intestinal. Algunos de ellos con patologías como malaria, tuberculosis y meningitis tuberculosa.

Las estancias hospitalarias se prolongan, ya que se egresa a estos niños con tratamientos completos, porque si no se hace así corre el riesgo de que no cumplan. Al egresar van a ir a un medio totalmente adverso: albergues donde habitan de 15 a 20 personas y la capacidad de estos es para 10 , solo el $22 \%$ cuentan con las condiciones adecuadas y algunos de ellos están declarados inhabitables.

Entre los meses de octubre del 2004 a febrero del 2005 se hospitalizó a 37 (4\% del total de los internamientos) niños indígenas, con un promedio de estancia hospitalaria de 9 días. Veinticinco (64\%) de estos casos con bronconeumonía y $16 \%$ con fallo respiratorio que requirió su traslado al Hospital Nacional de Niños. Este número elevado de casos con patología respiratoria aguda incrementó el porcentaje de ocupación del servicio a cifras superiores al $100 \%$. La bronconeumonía no sólo es uno de los diagnósticos más frecuentes de egreso sino que llevó a una intervención urgente y permanente del equipo de salud dada la severidad de los casos durante toda su estancia. Situación expuesta en el trabajo del grupo de investigadoras del INCIENSA, "Estacionalidad de egresos por neumonía en el servicio de Pediatría del Hospital Dr. Maximiliano Peralta y su asociación con la migración indígena Ngöbe Buglé", presentado en este número de la revista Acta Médica Costarricense.

Como resultado de esta investigación, la jefatura y los funcionarios del servicio de Pediatría gestionaron medidas operativas tendientes a mejorar la calidad de los registros y la definición de caso. Además, de continuar con lo establecido en las políticas de atención del servicio, la cual debe ser integral humanizada con calidad. Con el tiempo el servicio pretende determinar el impacto de estas acciones.

Por todo lo expuesto, los funcionarios del servicio de Pediatría instan a las autoridades de salud del país, a realizar una evaluación exhaustiva del impacto de la migración indígena en la salud pública costarricense, sin dejar de lado las medidas preventivas y la atención humanitaria del manejo de estos individuos.

Dra. María del Rosario Calvo Fonseca

Jefe de Pediatría

Hospital Dr. Maximiliano Peralta

\section{Referencias}

Abelardo Morales Gamboa. Programa de Migraciones Internacionales situación de los trabajadores migrantes en América Latina.

Alexander Jiménez Matarrita. Sociedades hospitalarias Costa Rica y la acogida de inmigrantes 2004.

3. Ministerio de Salud. Área de Salud de los Santos. Trabajo de Campo Plan de atención integral del inmigrante. Costa Rica: Ministerio de Salud 2002.

4. Banco Mundial. Panamá prioridades y estrategias para la reducción de la pobreza en Panamá 2000. 